\title{
Antideuteron limits on decaying dark matter with a tuned formation model
}

\author{
L. A. Dal and A. R. Raklev \\ Department of Physics, University of Oslo, Oslo N-0371, Norway
}

(Received 5 March 2014; published 5 May 2014)

\begin{abstract}
We investigate the production of antideuterons from decaying dark matter, using gravitinos in supersymmetric models with trilinear $R$-parity-violating (RPV) operators as an example. The model used for antideuteron formation is shown to induce large uncertainties in the predicted flux, comparable to uncertainties from cosmic-ray propagation models. We improve on the formation model by tuning hadronization and coalescence parameters in Monte Carlo simulations to better reproduce the hadron spectra relevant for antideuteron production. In light of current bounds on fluxes and future prospects from the AMS-02 and GAPS experiments we set limits on RPV couplings as a function of the gravitino mass.
\end{abstract}

DOI: $10.1103 /$ PhysRevD.89.103504

PACS numbers: 95.35.+d, 27.10.+h, 98.70.Sa, 12.60.Jv

\section{INTRODUCTION}

The last few years have seen a lot of interest in decaying dark matter scenarios, in which dark matter (DM) candidates are very long-lived particles. Having decaying rather than annihilating DM candidates leads to important consequences for indirect detection signals, as the flux of decay products scale with the density of dark matter, whereas the flux of annihilation products scales with the DM density squared. Such long-lived particles would be natural if the only coupling to Standard Model particles is very weak. Gravitational interaction is one such alternative.

In supersymmetric models, negative results from LHC searches for the standard missing energy signature of dark matter suggest looking at alternative models where $R$ parity is violated. With the gravitino as the lightest supersymmetric particle, the strength of the gravitational coupling means that gravitinos can naturally have lifetimes exceeding the age of the Universe. Such gravitino DM scenarios have been considered for some time now, beginning with Refs. [1-3], with specific indirect detection signatures of gamma rays, positrons, antiprotons and neutrinos.

With a well-motivated example of decaying dark matter, we will here investigate the production of antideuterons in gravitino decays for models with trilinear $R$-parityviolating (RPV) operators. Use of the antideuteron channel for the indirect detection of DM was first suggested in Ref. [4], and antideuterons have already been considered for generic decaying dark matter [5], and also specifically for gravitino DM [6] in models with bilinear RPVoperators. We note several important differences between the present paper and previous works. The generic DM study [5] includes only two-body decays with no baryon- or lepton-number violation, and therefore cannot describe the three-body decays of gravitinos into quarks and leptons due to trilinear RPV operators. The work on RPV gravitino decays [6] does take three-body RPV decays into account, but only for models with bilinear RPV operators. We shall see that the baryonnumber-violating trilinear couplings produce significantly more antideuterons than any other RPV decays previously considered. Moreover, we will use the resulting predicted antideuteron flux at the Earth to set limits on the trilinear RPV couplings, and unlike Ref. [5], we do not make the simplifying assumption of isotropic and uncorrelated distributions of antiprotons and antineutrons in the decays.

However, for readers interested in antideuteron limits for generic dark matter models, the most interesting novelty in this paper is probably the inclusion and treatment of uncertainties in the formation model for antideuterons, in particular hadronization effects. We investigate the effect of phenomenological hadronization parameters in the HERWIG++ Monte Carlo event generator [7,8] and the $p_{0}$ parameter of the coalescence model, and retune these to better describe the particular hadron spectra relevant for antideuteron production. We present a set of recommended parameters for further studies that result in an improved description of antideuteron formation with smaller and quantifiable uncertainties.

The trilinear RPV terms involved in the gravitino decays under study have the form

$$
W \sim \lambda_{i j k} L_{i} L_{j} \bar{E}_{k}+\lambda_{i j k}^{\prime} L_{i} Q_{j} \bar{D}_{k}+\lambda_{i j k}^{\prime \prime} \bar{U}_{i} \bar{D}_{j} \bar{D}_{k},
$$

in the superpotential. Here $\lambda_{i j k}, \lambda_{i j k}^{\prime}$ and $\lambda_{i j k}^{\prime \prime}$ are RPV couplings, $Q$ and $L$ are left-handed quark and lepton superfield doublets, respectively, while $\bar{E}, \bar{U}$ and $\bar{D}$ are the corresponding left-handed superfield singlets for leptons and up- and down-type quarks, respectively. The first two terms violate lepton number, while the third violates baryon number conservation. From gauge symmetry, $i \neq j$ for $\lambda_{i j k}$ and $j \neq k$ for $\lambda_{i j k}^{\prime \prime}$. For the present purpose of antideuteron production we are interested in the couplings that give antiquarks in gravitino decays: $\lambda^{\prime}$ and $\lambda^{\prime \prime}$.

While these operators, if allowed, can cause problems, most seriously with proton decay, completely removing them by introducing $R$ parity [9] by hand seems excessive and $a d$ hoc. In particular it is known that the proton can be protected from decay by up to dimension- 6 operators by 
introducing so-called trialities instead, disallowing either lepton- or baryon-number-violation terms in Eq. (1) [10-12].

Limits on trilinear couplings have been set on the basis of indirect detection experiments in the antiproton, positron and gamma-ray channels [3,13]. These are generically much stronger than other (collider) limits on RPV couplings, resting only on the assumption that the gravitinos constitute a significant fraction of the total DM. The limits' dependence on sparticle masses is dominated by the gravitino mass; the other sparticles are less important, unless there is a dramatic difference in scales compared to the gravitino mass [3]. ${ }^{1}$

As noted in Ref. [14], DM decay and annihilation involving final-state quark-antiquark pairs are in general already severely constrained by antiproton searches. However, we will see that the trilinear operators we investigate here have stronger bounds from antideuterons due to the possibility of a triple antiquark final state. Antideuterons from DM decay or annihilation are also expected to have a much flatter spectrum at low energies than those from the main background, namely cosmic raygas interactions. In conjunction with the extremely low level of background expected, this leads to even stronger limits on the trilinear couplings.

The present upper bound on the antideuteron flux that we use to set limits comes from the BESS balloon experiment [15], while the AMS-02 experiment running onboard the ISS [16] and the planned GAPS balloon experiment [17] are expected to deliver stronger bounds in the near future. We give our predictions for future limits given null results from these searches.

The starting point of the calculation of the expected cosmic-ray flux of antideuterons from gravitino decays is the decay width for gravitinos in the processes $\tilde{G} \rightarrow \nu_{i} d_{j} \bar{d}_{k}, \ell_{i}^{-} u_{j} \bar{d}_{k}$ and $\tilde{G} \rightarrow u_{i} d_{j} d_{k}$ found in Ref. [18]. The parton-level processes are showered and hadronized using the HERWIG++ 2.6.0 Monte Carlo event generator $[7,8]$. The production of antideuterons from the resulting antiprotons and antineutrons is described by the so-called coalescence model. The complete formation model, from parton level to antideuterons, will be discussed in more detail in Sec. II, including the uncertainties from the hadronization model used. The derived antideuteron spectrum is then propagated through the Galaxy, and the antideuteron flux at the Earth is found in Sec. III. In Sec. IV we then use this flux to set limits on the relevant trilinear RPV couplings, and make predictions for what limits can be set with future experiments.

\section{ANTIDEUTERON PRODUCTION}

The fusion of antiprotons and antineutrons into antideuterons is commonly described by the coalescence

\footnotetext{
${ }^{1}$ For the purposes of this paper we set all other sparticle masses to $1 \mathrm{TeV}$, just outside the current reach of the LHC.
}

model. In this model, any $\bar{p} \bar{n}$ pair with a momentum difference $\Delta p<p_{0}$, for some chosen value of $p_{0}$, will fuse to produce an antideuteron. In our calculation, this condition is evaluated in the center-of-momentum frame of the individual $\bar{p}-\bar{n}$ pairs. In the limit $m_{\bar{p}}=m_{\bar{n}}$, this is equivalent to the condition on invariant relativistic momentum used in e.g. Ref. [14].

Traditionally, the coalescence prescription has been applied to the averaged energy spectra of the antinucleons from a process, but this approach is based on the assumption that the antinucleon spectra are isotropic and uncorrelated. These assumptions have been found not to hold in general in DM decay/annihilation processes $[19,20]$, and the results presented here are therefore generated by applying the coalescence prescription on a per-event basis.

\section{A. Current coalescence models}

The coalescence momentum $p_{0}$ can be found by running simulations of a particular process and fitting $p_{0}$ so that the result matches available experimental data. In principle, an uncertainty in the $p_{0}$ value can then be extracted and propagated to antideuteron fluxes. A wide range of $p_{0}$ values have previously been used in the literature; however, the majority of these were found using the suboptimal isotropic assumption.

One of the major difficulties in reducing errors is the lack of data on antideuteron production at colliders to constrain models. In Refs. [19-21] $p_{0}$ was calibrated against the single ALEPH antideuteron data point from hadron production at the $Z$ resonance [22], using per-event coalescence in HERWIG++ and PYTHIA $8[23,24]$. The resulting values were $p_{0}=110 \mathrm{MeV}$ for HERWIG++ and $p_{0}=$ $162 \mathrm{MeV}$ for PYTHIA, giving very different antideuteron spectra and indicating large model uncertainties of the order of a factor of 2-4 in the observable flux [20].

Recently, the authors of Ref. [14] have pointed out that antinucleons produced in weak decays should not participate in antideuteron formation, as they would be produced too far away from the primary vertex to interact with antinucleons produced in hadronization or other decays. This reduces the number of antinucleons available for antideuteron formation substantially, ${ }^{2}$ and the values of the coalescence momenta required to match experimental data become correspondingly higher. In Ref. [14] $p_{0}$ was calculated for several experimental data sets using perevent coalescence in PYTHIA, and best-fit $p_{0}$ values are found in the range 133-236 MeV. In Table I we compare these results to our own results with HERWIG++ for the same experiments using the default hadronization parameters.

\footnotetext{
${ }^{2}$ In $e^{+} e^{-}$collisions at the $Z$ resonance, we find the antinucleon production rate to be reduced by a factor $\sim 1.5$, leading to a reduction in the number of antideuterons produced for a given value of $p_{0}$ by a factor $\sim 2-3$.
} 
TABLE I. Best-fit values of $p_{0}$ in $\mathrm{MeV}$ for various experiments and event generators. Values for PYTHIA are taken from Ref. [14]. Note that not all processes are available in PYTHIA 8.

\begin{tabular}{lcccc}
\hline \hline Experiment & Process & PYTHIA 6 & PYTHIA 8 & HERWIG++ \\
\hline ALEPH [22] & $e^{+} e^{-}$ & $\ldots$ & 192 & 159 \\
CLEO [25] & $e^{+} e^{-}$ & $\ldots$ & 133 & 145 \\
ZEUS [26] & $e p$ & 236 & $\ldots$ & 150 \\
CERN ISR [27, 28] & $p p$ & $\ldots$ & 152 & 221 \\
ALICE [29] & $p p$ & 230 & $\ldots$ & 154 \\
\hline \hline
\end{tabular}

The experiments where data are available are, as mentioned, $e^{+} e^{-}$interactions at ALEPH, production from $\Upsilon(1 S)$ decay at CLEO [25], production in $e p$ scattering at $\sqrt{s}=318 \mathrm{GeV}$ in ZEUS [26], $p p$ scattering at $\sqrt{s}=$ $53 \mathrm{GeV}$ at the CERN ISR [27,28] and $p p$ scattering at $\sqrt{s}=7 \mathrm{TeV}$ in ALICE [29]. We note a smaller spread in the fits using HERWIG++, with the exception of the CERN ISR data.

As discussed in Ref. [20], the antideuteron production is quite sensitive to the hadronization procedure, and it is therefore unlikely that the best fit $p_{0}$ values found by Ref. [14] using versions of Pythia will be representative for events generated using HERWIG++. Differences between the Monte Carlo generators in Table I indicate their different modelings of the underlying physics.

An interesting question that arises is whether the coalescence model should be sensitive to the process in which the coalescing antinucleons were produced. If not, one would a priori expect the different experiments to be more or less consistent with a common value of the coalescence momentum $p_{0}$. This is not immediately clear in Table I for either event generator, although, as we shall see, the differences may be due to other parameters in the hadronization model. For an indication of whether the model is consistent across experiments we have performed a combined least-squares fit of $p_{0}$ over all the experiments for HERWIG++ using its default settings. This gives a best-fit $p_{0}=152 \mathrm{MeV}$ with $\chi^{2}=53.2$ for a total of 25 degrees of freedom (d.o.f.). The individual contributions to the $\chi^{2}$ are shown in Table II, where we also show for comparison the $\chi^{2}$ for fits to the individual experiments.

It is clear from Table II that the individual experiments can be well described, but not when combined. One

TABLE II. $\chi^{2}$ contribution from the various experiments for the individual best-fit values of $p_{0}$ and for the combined best-fit value of $p_{0}=152 \mathrm{MeV}$.

\begin{tabular}{lccc}
\hline \hline Experiment & $\chi^{2}$, best-fit $p_{0}$ & $\chi^{2}, p_{0}=152 \mathrm{MeV}$ & $N_{\text {bins }}$ \\
\hline ALEPH & 0.0 & 0.2 & 1 \\
CLEO & 7.6 & 10.5 & 5 \\
ZEUS & 3.7 & 3.8 & 3 \\
CERN ISR & 5.0 & 33.2 & $4+4$ \\
ALICE & 5.1 & 5.5 & 9 \\
\hline \hline
\end{tabular}

possible reason behind this failure is the choices made in the necessary phenomenological tuning of Monte Carlo event generators in order to reproduce data. Event generators such as HERWIG++ and PYTHIA are typically tuned with emphasis on particle multiplicities, and very little or no emphasis at all with respect to two-particle correlations. A systematic spread in the best-fit value of $p_{0}$ between processes and energies is therefore to be expected because of the emphasis the various processes/experiments have been given in tuning, which in turn influences the predicted production of the antiproton and antineutron building blocks of the antideuteron.

The failure to describe data with statistical fluctuations means that the spread of $p_{0}$ values we have found are not a good measure of the uncertainty in the antideuteron flux coming from the coalescence model. The authors of Ref. [30] have demonstrated how retuning hadronization parameters can be used to improve the physics description for a particular process (Higgs production), and to determine the underlying uncertainty from the tuning of these parameters. In the following section we will proceed by retuning a selection of the HERWIG++ hadronization parameters in order to be able to simulate antideuteron production more consistently.

\section{B. Tuning of the formation model}

\section{Choice of parameters}

The full set of parameters in the HERWIG++ hadronization model constitutes a rather big parameter space, and a full parameter scan is not realistic with the large statistics required for per-event coalescence deuteron production. In addition to the coalescence momentum $p_{0}$, we therefore restrict ourselves to tuning the three parameters studied in Ref. [20] that were found to individually have the strongest impact on antideuteron production: ${ }^{3}$ PWTDIQUARK, ClMAxLight and PSPlitLight.

In order to make the role of these parameters clear, we briefly review the HERWIG++ cluster hadronization model. Following the perturbative QCD cascade, all gluons are first split into quark-antiquark pairs. Color-connected (di) quark pairs are subsequently combined to form colorsinglet clusters. Light clusters are decayed into hadrons, while clusters heavier than some mass threshold are iteratively fissioned into lighter clusters. The CLMAXLight parameter is one of two parameters involved in specifying this mass threshold for clusters consisting of light quarks $(u, d$ or $s)$. The criterion for fission is that the cluster mass $M$ fulfills

$$
M^{p} \geq m_{C}^{p}+\left(m_{1}+m_{2}\right)^{p},
$$

\footnotetext{
${ }^{3}$ We do not include the parameter AlPHAMZ, the strong coupling at the $Z$ mass, which is constrained by high-energy scattering data.
} 
where $m_{1}$ and $m_{2}$ are the masses of the two partons in the cluster, $m_{C}$ is CLMAXLIGHT and $p$ is the other parameter involved. Changing CLMAXLight will affect the masses available in cluster decay and as a result the probability to create (anti)baryons.

For clusters above this threshold, a light quark-antiquark pair is popped from the vacuum, and two clusters are formed with one of the new and one of the original partons in each cluster. The inverse of PSPLITLight is a power that controls the mass distribution of the resulting clusters when they contain only light quarks.

The final step of the hadronization process is cluster decay. Given a cluster of flavor $\left(q_{1}, \bar{q}_{2}\right)$, a (di)quark-anti(di) quark pair $(q, \bar{q})$ or $(q q, \bar{q} \bar{q})$ is extracted from the vacuum, and two hadrons with the flavors $q_{1} \bar{q}$ and $q \bar{q}_{2}$, or $q_{1} q q$ and $\bar{q} \bar{q} \bar{q}_{2}$, are formed. The PWTDIQUARK parameter controls the probability of choosing a diquark pair to be popped from the vacuum in this process, and thus directly controls the probability of creating baryons.

Hadronization parameters will in general affect both (anti)nucleon yields through the cluster masses and their two-particle correlations. For the parameters considered here, we have found that this holds true for CLMAXLigHT and PSplitLight, while PwTDIQUARK appears to mainly affect the (anti)nucleon yields, which is to be expected since it controls the production of baryon-antibaryon pairs, as opposed to baryon or antibaryon pairs.

\section{Tuning procedure}

For the tuning of the formation model we use a leastsquares fit, minimizing the quantity

$$
S=\sum_{i}^{\text {bins, experiments }}\left(\frac{y_{i}^{\exp }-y_{i}^{\mathrm{MC}}\left(\alpha_{j}\right)}{\sigma_{i}}\right)^{2},
$$

where $y_{i}^{\exp }$ and $y_{i}^{\mathrm{MC}}$ are the experimental and Monte Carlo values for bin $i$, respectively, $\alpha_{j}$ are the four parameters being tuned, and the sum is over all experimental data bins. In the fit we use all the available experimental data listed in Sec. II A except the $p p$ data, and we also include data on proton production in $e^{+} e^{-}$collisions (see below). Our reason for not including the $p p$ data is twofold. Firstly, generating sufficient statistics for these experiments is highly computationally expensive. Including CERN ISR and ALICE in our fit would increase the required CPU time by a factor of 6 , and is thus not feasible in this context. Secondly, the extremely large rise in the $\chi^{2}$ contribution from the CERN ISR data between the individual and the joint fit seen in Table II might indicate some process dependence in the coalescence model-even if this is not seen in the ALICE data-or that there might be some problem with the CERN ISR data or our interpretation of it.

The uncertainty $\sigma_{i}^{2}=\sigma_{i, \mathrm{MC}}^{2}+\sigma_{i, \exp }^{2}$ used for bin $i$ is given by $\sigma_{i, \mathrm{MC}}$ and $\sigma_{i, \exp }$, the Monte Carlo and experimental uncertainties of the corresponding bin, respectively. Ideally, one should generate enough events for the Monte Carlo uncertainty to be negligible compared to the experimental one, but for antideuterons, this is not practical in the context of a parameter scan due to the very low production rate. We therefore compromise by generating 100 times the number of events needed for the Monte Carlo uncertainty to match the experimental uncertainty when averaged over all bins.

In order to avoid tuning into unrealistic spectra for antiprotons and antineutrons - the antideuteron building blocks-we constrain the fit further by using antiprotons in the tune. We use antiproton data from the LEP experiments OPAL [31] and ALEPH [32]. The OPAL data constitute half of the light baryon data used in tuning HERWIG++, the other half being proton production at similar energies in $e^{+} e^{-}$collisions from SLD. Because of the large number of data points available (26 antiproton data points from ALEPH alone compared to 9 antideuteron data points from ALEPH, CLEO and ZEUS combined) we reweight the antiproton data in the fit with a factor $1 / 25$ to keep it from dominating the parameter determination.

The antiproton data points of the two experiments are known to not be compatible at very high antiproton momenta, and should therefore not be used together directly. We combine the data of the two experiments using a weighted average

$$
y_{i}=\left(\frac{y_{i, A}}{\sigma_{i, A}^{2}}+\frac{y_{i, O}}{\sigma_{i, O}^{2}}\right)\left(\frac{1}{\sigma_{i, A}^{2}}+\frac{1}{\sigma_{i, O}^{2}}\right)^{-1},
$$

where the $A$ and $O$ subscripts denote ALEPH and OPAL, respectively. We combine the experimental uncertainties accordingly, and use the differences in central values $\Delta y_{i}=\left|y_{\mathrm{ALEPH}}-y_{\mathrm{OPAL}}\right|$ as an additional systematic error describing our ignorance of the reason for the incompatible data, giving a combined uncertainty of

$$
\sigma_{i}=\sqrt{\left(\frac{1}{\sigma_{i, A}^{2}}+\frac{1}{\sigma_{i, O}^{2}}\right)^{-1}+\left(\Delta y_{i}\right)^{2}} .
$$

As the energy bins of the two experiments do not match, we choose the binning of the ALEPH data set, and interpolate the OPAL data correspondingly.

For the minimization procedure we use the MIGRAD algorithm in MINUIT [33]. However, due to the per-event coalescence model used, it is prohibitively expensive in terms of computation time to directly evaluate the $\chi^{2}$ as many times as minimization requires (one parameter point takes $\sim 120$ CPU core hours on a modern processor). Instead, we sample parameter points on grids, and use so-called RBFs—see e.g. Ref. [34] and further discussion below-to approximate the shape of the function between the sampled points. The global minimum is then found through an iterative procedure. First, we apply the MIGRAD algorithm on the approximated function, starting 
TABLE III. Step sizes and fully covered parameter ranges for the grid scans. $p_{0}$ values are in units of $\mathrm{MeV}$.

\begin{tabular}{lccc}
\hline \hline Parameter & Step size & Range, grid 1 & Range, grid 2 \\
\hline$p_{0}$ & 10 & $110-200$ & $110-200$ \\
CLMAXLIGHT & 1.33 & $2.00-7.33$ & $1.33-5.33$ \\
PSPLITLIGHT & 0.53 & $0.15-2.82$ & $0.42-2.02$ \\
PwTDIQUARK & 0.117 & $0.250-0.717$ & $0.075-0.775$ \\
\hline \hline
\end{tabular}

in the best point sampled, to find a temporary global minimum. We then evaluate this point with HERWIG++ to find the true function value. If the difference between the true and the approximate function value at the minimum is greater than a chosen value, the RBFs are recalculated with the newly evaluated point included, and the procedure is repeated. We here accept the minimum if the difference between the true and the approximate function value is less than $\sim 0.5$, which is roughly the typical size of the errors in $S$ in our calculation due to limited statistics.

In the procedure to set up the RBFs, we sample an initial total of 342 points in the three HERWIG++ hadronization parameters in two grids with equal step sizes, shifted a half step with respect to each other in each parameter direction. For each point in the other three parameters, we calculate the antideuteron flux for $p_{0}$ values in steps of $1 \mathrm{MeV}$ from 5 to $400 \mathrm{MeV}$; this can be done per event and is computationally inexpensive. In the radial basis functions, however, we use only ten different values of $p_{0}$ per point in the other parameters. We do this to avoid bias from having a much larger number of samples in one parameter, and also because the calculation becomes highly memory intensive with a large number of points.

The step sizes and ranges fully covered by the two grids are listed in Table III. We have also sampled regions outside the quoted ranges to investigate the possible existence of other global minima. Note that not all regions of the parameter space are covered by both grids, but overlap between the grids is ensured in regions where the function value is low enough to allow for a possible global minimum.

\section{Radial basis functions}

A commonly used method for approximating function values between scattered multivariate data points is RBFs; for a reference see Ref. [34]. Given a function $f(x)$ whose value is known at a set of distinct points $x_{i}$, the goal is to construct an approximated function $s(x)$, such that

$$
s\left(x_{i}\right)=f\left(x_{i}\right) .
$$

This can be done through a linear combination of radially symmetric functions $\phi(r)$ centered in $x_{i}$,

$$
s(x)=\sum_{i} a_{i} \phi\left(\left\|x-x_{i}\right\|\right) .
$$

The coefficients $a_{i}$ are found by imposing Eq. (6), and for the choices of $\phi(r)$ mentioned below, the solution is unique [34]. The functions $\phi(r)$ are referred to as radial basis functions, and popular choices include

$$
\phi(r)= \begin{cases}r & \text { linear, } \\ \exp \left(-c^{2} r^{2}\right) & \text { Gaussian, } \\ \sqrt{r^{2}+c^{2}} & \text { multiquadratic. }\end{cases}
$$

The two latter choices introduce a free parameter, $c$, that will affect the shape of $s(x)$.

For this work, we tested all of the RBF choices mentioned above, and found linear RBFs to be the best option. We found that Gaussian and multiquadratic RBFs generally gave sharper minima than linear RBFs, but the widths of the minima depended significantly on the shape parameter $c$. With no free parameters and somewhat wider minima, linear RBFs will thus give a less ambiguous and more conservative estimate of the parameter errors.

Since the RBFs are radially symmetric, the interpolation between points will depend on the normalization of the different parameters that we are trying to minimize over. The divergence of $\phi(r)$ is radially symmetric in the parameter space, but the data was sampled with different step sizes in the different parameter directions. This means that the relative divergence is much larger in parameters with small step sizes than in parameters with large step sizes. To avoid having any preferred directions in the interpolation, we therefore normalize the parameters such that the step sizes are equal in all parameter directions.

\section{Results}

In Table IV we show the resulting values of the parameters at the minimum found for the $\chi^{2}, \chi_{\min }^{2}=$ 10.6 for effectively 14.2 d.o.f. due to the down-weighting of the proton data, and the $1 \sigma$ error bands determined from the shape of the $\chi^{2}$ distribution.

\section{Antideuteron spectra}

Using the improved formation model and the uncertainties determined for its parameters in the previous section we now calculate the antideuteron spectra from gravitino decays.

TABLE IV. Results from the hadronization parameter fit compared to default values in HERWIG++. $p_{0}$ values are in units of $\mathrm{MeV}$.

\begin{tabular}{lccc}
\hline \hline Parameter & Default value & Value at $\chi_{\min }^{2}$ & Uncertainty $^{4}$ \\
\hline$p_{0}$ & $\ldots$ & 143.2 & ${ }_{-5.5}^{+6.2}$ \\
CLMAXLiGHT & 3.25 & 3.03 & ${ }_{-0.15}^{+0.18}$ \\
PSPLITLIGHT & 1.20 & 1.31 & ${ }_{-0.32}^{+0.19}$ \\
PwTDIQUARK & 0.49 & 0.48 & ${ }_{-0.04}^{+0.15}$ \\
\hline \hline
\end{tabular}




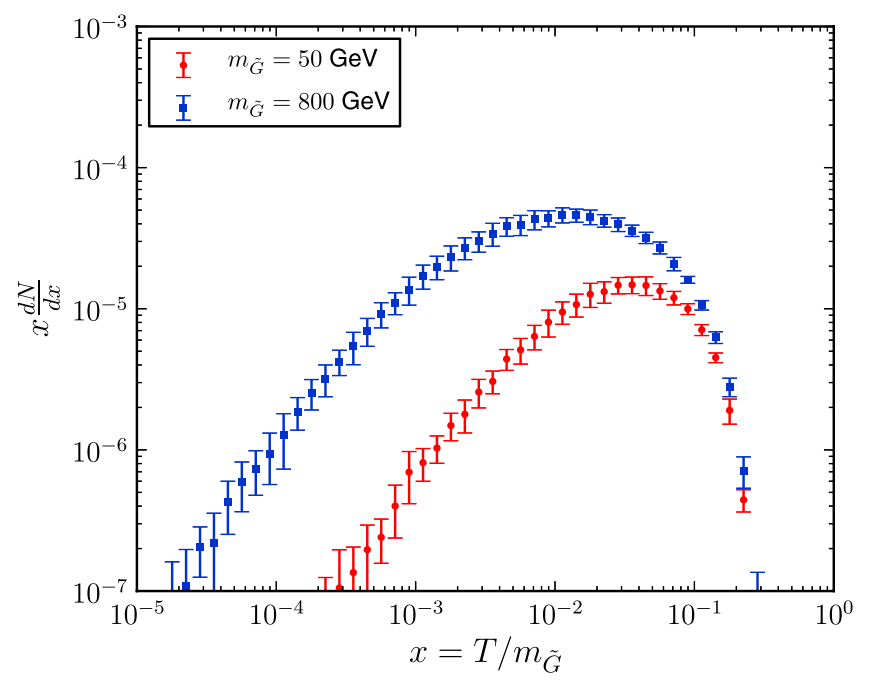

FIG. 1 (color online). Antideuteron spectra from gravitino decay through the $L_{1} Q_{1} \bar{D}_{2}$ operator for gravitino masses of $50 \mathrm{GeV}$ and $800 \mathrm{GeV}$. For both masses $\lambda=1$ is used; the normalization of the spectra scales as $\lambda^{2}$.

We simulate a representative selection of trilinear RPV couplings and gravitino masses with potential final-state antiquarks, both heavy and light antiquarks, in the single dominant coupling approximation: $\lambda_{112}^{\prime}, \lambda_{133}^{\prime}, \lambda_{112}^{\prime \prime}$ and $\lambda_{323}^{\prime \prime}$. Our expectation is that the $\lambda_{112}^{\prime \prime}$ coupling with its three light (anti)quarks will have the greatest (anti)deuteron production.

In Figs. 1 and 2 we show the resulting antideuteron production spectrum for a selection of couplings and masses in terms of the scaled kinetic energy $x \equiv T / m_{\tilde{G}}$. The $1 \sigma$ error bars shown are determined by sampling ten

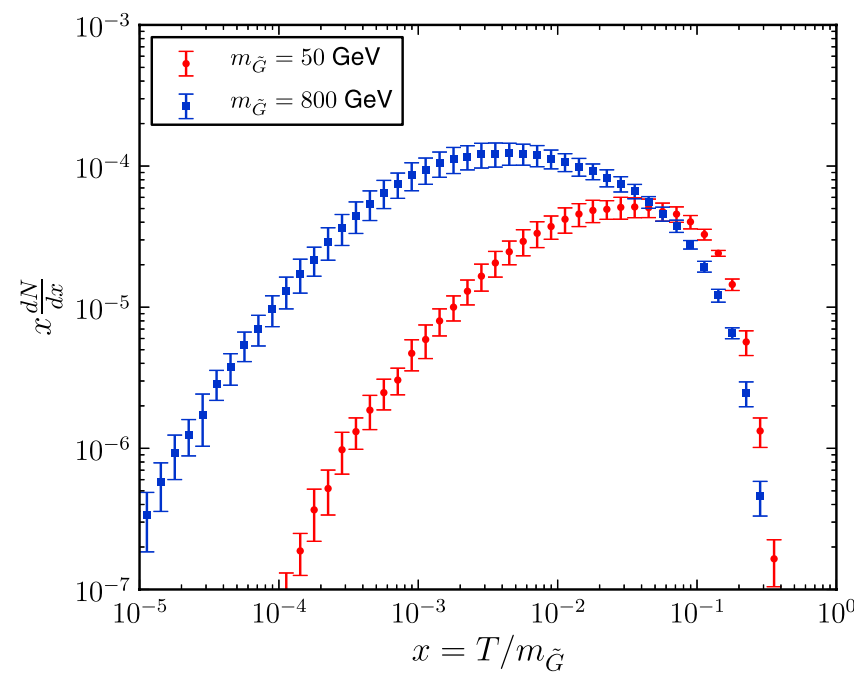

FIG. 2 (color online). Antideuteron spectra from gravitino decay through the $\bar{U}_{1} \bar{D}_{1} \bar{D}_{2}$ operator for gravitino masses of $50 \mathrm{GeV}$ and $800 \mathrm{GeV}$. For both masses $\lambda=1$ is used; the normalization of the spectra scales as $\lambda^{2}$. sets of hadronization parameter values from a likelihood function constructed on the basis of the $\chi^{2}$ distribution found in the previous section. For each set of parameter values the antideuteron spectrum was calculated using $10^{8}$ events. As a result, the statistical uncertainties on the spectra are low compared to the uncertainty inferred from the parameter likelihood in all but the low-energy ends of the spectra where the antideuteron yield is low.

As expected, decays through the $\bar{U}_{1} \bar{D}_{1} \bar{D}_{2}$ operator give a higher antideuteron yield than decays through $L_{1} Q_{1} \bar{D}_{2}$, but by less than an order of magnitude. We find that the uncertainty in the spectrum contributed by $p_{0}$ and the investigated hadronization parameters to be a factor $\sim 1.3$ for most energies. This is substantially lower than the uncertainty from $p_{0}$ alone found in Ref. [14] using various versions of PYTHIA with default settings.

\section{ANTIDEUTERON FLUX AT THE EARTH}

\section{A. Astrophysical background}

One of the strengths of the antideuteron channel in DM searches is the very low expected astrophysical background flux. Previous estimates of the background flux have been done by applying the coalescence model to antinucleon spectra under the assumption of isotropic antinucleon spectra. More recently, however, a recalculation of the astrophysical antideuteron flux was performed in Ref. [35] using the more correct per-event coalescence in Monte Carlo event generators. This leads to an expected antideuteron background that is a factor $\sim 2$ lower than the previous estimates. The antideuteron background spectra used in this work are the recalculated ones of Ref. [35].

\section{B. Gravitino decay signal}

\section{Propagation of antideuterons}

In order to find the resulting antideuteron flux at the Earth, the antideuterons must be propagated through the Galaxy from their point of origin in the DM halo. Charged particles propagating through the Galaxy scatter on fluctuations in the turbulent magnetic field, leading to a randomwalk behavior. This movement is well described using a diffusion approximation, and here we use the so-called twozone propagation model. This is a cylindrical diffusion model consisting of a magnetic halo of radius $R=20 \mathrm{kpc}$ and half-height $L$ (a free parameter), and a thin gaseous disk of the same radius and a half-height of $h=100 \mathrm{pc}$.

Assuming steady-state conditions, and neglecting reacceleration and nonannihilating inelastic scattering, the diffusion equation describing this model is given by

\footnotetext{
${ }^{4}$ The $\chi^{2}$ around the minimum is highly nonparabolic, and the uncertainty was therefore calculated using the MINOS algorithm in MINUIT [33]. This typically gives larger values for the uncertainties than calculating uncertainties from the error matrix.
} 


$$
-D(T) \nabla^{2} f+\frac{\partial}{\partial z}\left(\operatorname{sign}(z) f V_{c}\right)=Q-2 \mathrm{~h} \delta(z) \Gamma_{\mathrm{ann}}(T) f,
$$

where $f(\boldsymbol{x}, T)=d N_{\bar{d}} / d T$ is the number density of antideuterons per unit kinetic energy, $D(T)=D_{0} \beta \mathcal{R}^{\delta}$ is the (spatial) diffusion coefficient, $V_{c}$ is a convective wind perpendicular to the Galactic disk, $z$ is the vertical coordinate, $\beta=v / c$ is the velocity and $\mathcal{R}$ is the rigidity of antideuterons in GV. Here $\delta, D_{0}$, and $V_{c}$ are free parameters.

The annihilation rate, $\Gamma_{\text {ann }}$, of antideuterons on interstellar gas in the Galactic disk is given by

$$
\Gamma_{\mathrm{ann}}=\left(n_{H}+4^{\frac{2}{3}} n_{\mathrm{He}}\right)\left\langle\sigma_{\bar{d} p}^{\mathrm{ann}} v\right\rangle,
$$

where we use the values $n_{H} \approx 1 \mathrm{~cm}^{-3}$ and $n_{\mathrm{He}} \approx 0.07 n_{H}$ for the number densities of $\mathrm{H}$ and $\mathrm{He}$ in the disc, respectively, and where the factor $4^{\frac{2}{3}}$ accounts for the difference in annihilation cross section between $\mathrm{H}$ and $\mathrm{He}$, assuming simple geometrical scaling. For the annihilation cross section, we use a fit to experimental data [36,37], as seen in Fig. 3.

The source term, $Q$, is determined by the dark matter density profile of our Galaxy. Most of the commonly used profiles can be parametrized as

$$
\rho(r)=\frac{\rho_{0}}{(r / a)^{\gamma}\left[1+(r / a)^{\alpha}\right]^{(\beta-\gamma) / \alpha}},
$$

where $a$ and $\rho_{0}$ are a characteristic length and a characteristic density, respectively, while $\alpha, \beta$ and $\gamma$ are dimensionless parameters. The Navarro-Frenk-White (NFW) profile [38] is given by $\alpha=1, \beta=3, \gamma=1$.

For the free parameters in the model we adopt the three sets of values found in Ref. [39] to yield maximal, median

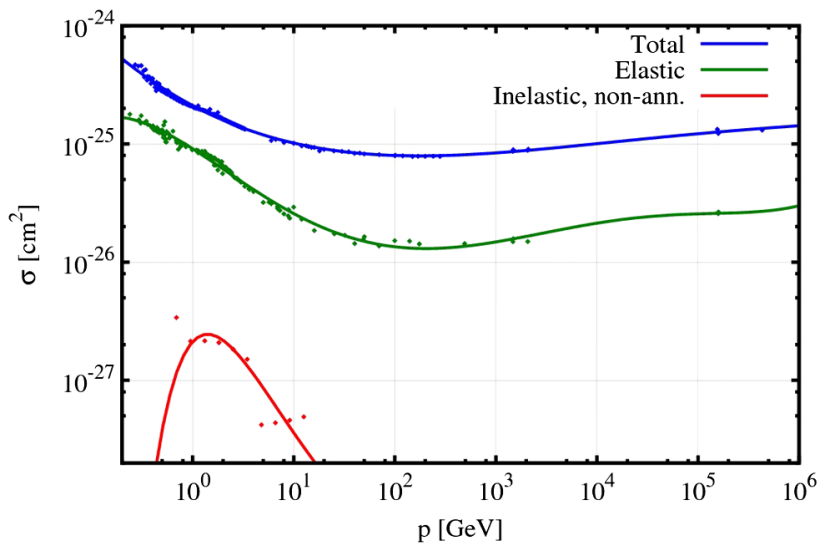

FIG. 3 (color online). Cross-section data for antideuterons on interstellar protons as a function of the antideuteron momentum. The points indicate experimental data, while the lines show the fits to the data that were used in our calculations. The lower line shows the inelastic nonannihilating cross-section, the middle line shows the elastic cross-section, while the upper line shows the total cross-section. and minimal antiproton fluxes from DM annihilations, while being compatible with the observed $\mathrm{B} / \mathrm{C}$ ratio. These parameter sets are labeled "max," "med" and "min," respectively, and their values are listed in Table V.

The diffusion equation (9) can be solved (semi)analytically [40], giving an antideuteron flux at the position of the Earth for decaying DM [5],

$$
\Phi_{\bar{d}}\left(T, r_{\odot}\right)=\frac{v_{\bar{d}}}{4 \pi} \frac{1}{\tau} \frac{\rho_{\odot}}{M_{\mathrm{DM}}} R(T) \frac{d N_{\bar{d}}}{d T},
$$

where

$$
\begin{aligned}
R(T)=\sum_{n=1}^{\infty} J_{0}( & \left.\zeta_{n} \frac{r_{\odot}}{R}\right) \exp \left(-\frac{V_{c} L}{2 K}\right) \frac{y_{n}(L)}{A_{n} \sinh \left(S_{n} L / 2\right)} \\
y_{n}(Z)= & \frac{4}{J_{1}^{2}\left(\zeta_{n}\right) R^{2}} \int_{0}^{R} \mathrm{~d} r r J_{0}\left(\frac{\zeta_{n} r}{R}\right) \\
& \times \int_{0}^{Z} \mathrm{~d} z\left\{\exp \left(\frac{V_{c}(Z-z)}{2 D}\right)\right. \\
& \left.\times \sinh \left(\frac{S_{n}(Z-z)}{2}\right) \frac{\rho(r, z)}{\rho_{\odot}}\right\} \\
A_{n}=2 h & \Gamma_{\mathrm{ann}}+V_{c}+D S_{n} \operatorname{coth}\left(S_{n} L / 2\right)
\end{aligned}
$$

and

$$
S_{n}=\sqrt{\frac{V_{c}^{2}}{D^{2}}+4 \frac{\zeta_{n}^{2}}{R^{2}}}
$$

The function $R(T)$ encodes the astrophysics of the propagation, and is completely independent of the particle physics of the DM decay. The function can thus be precalculated for any given set of propagation parameters and halo model, and subsequently used for any DM candidate decaying into antideuterons. Solar modulations are further taken into account by replacing the final kinetic energy of the particles $T$ with a modified kinetic energy near the Earth [41], $T_{\otimes}=T-|Z e| \phi_{\text {Fisk }}$, where the socalled Fisk potential $\phi_{\text {Fisk }}=0.5 \mathrm{GV}$ is an effective potential that parametrizes the energy loss from the solar wind. The corresponding antideuteron flux near the Earth is then given by

TABLE V. Propagation parameters for the max, med and min models.

\begin{tabular}{lclcc}
\hline \hline Model & $L$ in kpc & \multicolumn{1}{c}{$\delta$} & $D_{0}$ in $\mathrm{kpc}^{2} \mathrm{Myr}^{-1}$ & $V_{c}$ in $\mathrm{km} \mathrm{s}^{-1}$ \\
\hline $\max$ & 15 & 0.46 & 0.0765 & 5 \\
$\operatorname{med}$ & 4 & 0.7 & 0.0112 & 12 \\
$\min$ & 1 & 0.85 & 0.0016 & 13.5 \\
\hline \hline
\end{tabular}




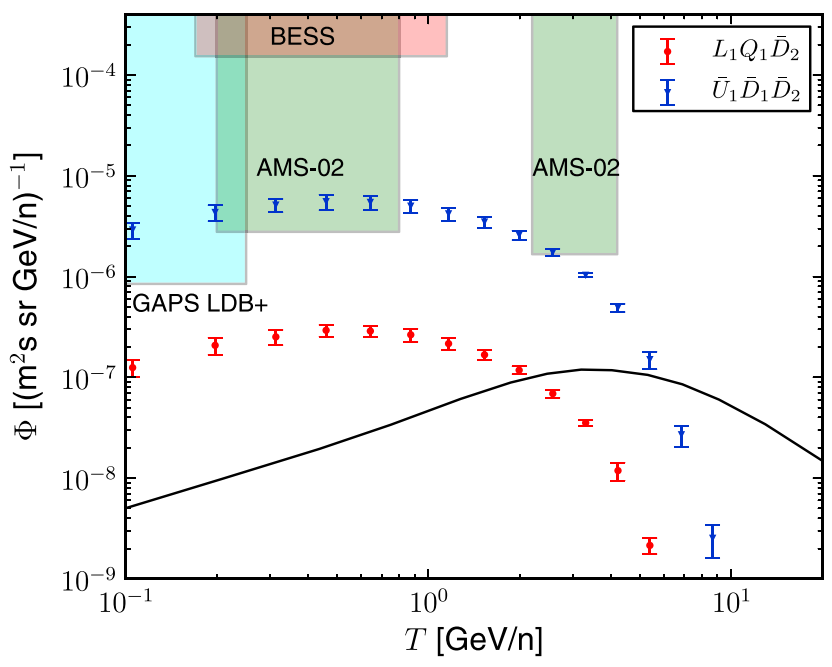

FIG. 4 (color online). Antideuteron flux at the Earth from gravitino decays with $m_{\tilde{G}}=50 \mathrm{GeV}$ for the NFW DM halo profile and "med" propagation parameters. The colors show different RPV couplings. For all couplings $\lambda=10^{-5}$ is used; the normalization of the spectra scales as $\lambda^{2}$. The solid black line shows the expected background flux.

$$
\Phi_{\otimes}=\frac{p_{\otimes}^{2}}{p^{2}} \Phi=\frac{2 m_{\bar{d}} T_{\otimes}+T_{\otimes}^{2}}{2 m_{\bar{d}} T+T^{2}} \Phi .
$$

With this diffusion model, solar modulation, and the antideuteron spectra calculated above, we show the resulting flux at the Earth for our choice of models in Figs. 4 and 5. The error bands shown are determined as in Sec. II C. RPV operators with similar flavor contents give

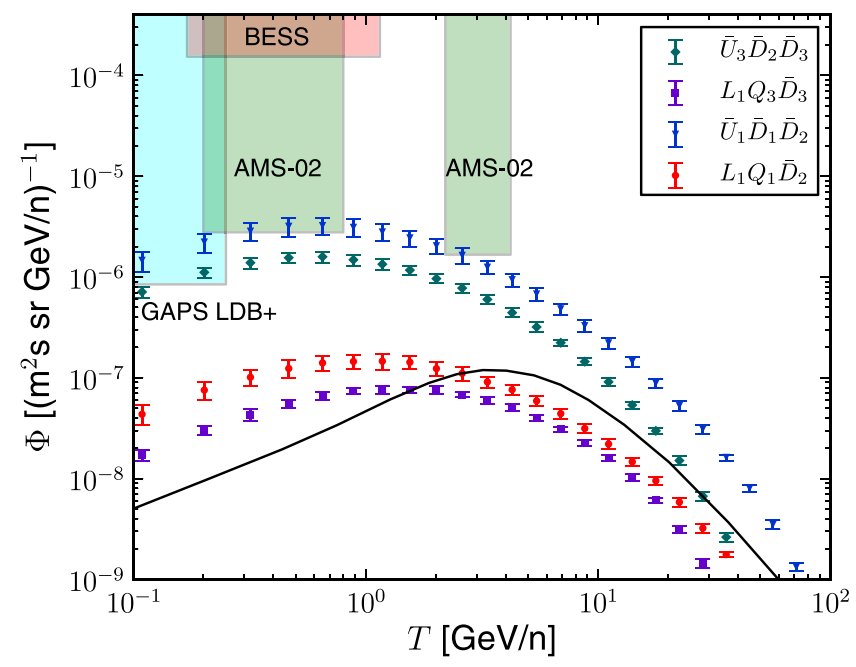

FIG. 5 (color online). Antideuteron flux at the Earth from gravitino decays with $m_{\tilde{G}}=800 \mathrm{GeV}$ for the NFW DM halo profile and "med" propagation parameters. The colors show different RPV couplings. For all couplings $\lambda=10^{-9}$ is used; the normalization of the spectra scales as $\lambda^{2}$. The solid black line shows the expected background flux. similar fluxes, and are thus omitted here. An example is the $L_{1} Q_{2} \bar{D}_{2}$ operator, which yields a similar flux as the $L_{1} Q_{1} \bar{D}_{2}$ operator.

The BESS, AMS-02 and GAPS limits shown are (prospective) $95 \%$ C.L. exclusion limits ${ }^{5}$ calculated using the $C L_{s}$ method, as discussed in Sec. IV. We note that the fluxes near the Earth from $L Q \bar{D}$ and $\bar{U} \bar{D} \bar{D}$ operators differ by an additional half order of magnitude compared to the corresponding source spectra seen in Figs. 1 and 2. This additional half order of magnitude comes from a corresponding difference in the gravitino lifetime.

\section{Antideuteron detection experiments}

In order to set exclusion limits on the antideuteron flux and the RPV couplings, we need to be able to calculate the number of expected events at the various experiments that search for antideuterons. These calculations will be described in the present section.

\section{BESS}

In the calculation of their limit on the cosmic-ray antideuteron flux, the BESS collaboration uses the estimate [15]

$$
\Phi_{\text {max }}=\frac{N_{\text {obs }}}{\left|S \Omega \mathcal{E}_{\text {tot }}\left(1-\delta_{\text {sys }}\right)\right|_{\text {min }} t_{\text {live }}\left(T_{2}-T_{1}\right)},
$$

where $N_{\text {obs }}$ is the number of observed events, $S \Omega$ is the geometrical acceptance, $\mathcal{E}_{\text {tot }}$ is the total detection efficiency, $\delta_{\text {sys }}$ is the total systematic uncertainty, $t_{\text {live }}$ is the live time of the experiment, and $\left(T_{1}, T_{2}\right)$ is the kinetic energy range in which the flux is being measured. The "min" subscript indicates that the minimal value of the quantity, as a function of energy, should be used to obtain a conservative estimate for the flux. This is also the reason for including the systematic uncertainty in the equation. The corresponding equation for calculating the expected number of events for a given flux is

$$
N=\int_{T_{1}}^{T_{2}} S \Omega \mathcal{E}_{\text {tot }}\left(1-\delta_{\text {sys }}\right) t_{\text {live }} \Phi d T .
$$

For BESS the relevant experimental energy range is $T_{1}=0.17 \mathrm{GeV} / \mathrm{n}$ and $T_{2}=1.15 \mathrm{GeV} / \mathrm{n}$ and the total systematic uncertainty was estimated by the experiment to lie at the $10 \%$ level, $\delta_{\text {sys }}=0.1$. We take the effective exposure $S \Omega \mathcal{E}_{\text {tot }} t_{\text {live }}$, as a function of energy, from Fig. 2 in Ref. [15].

\footnotetext{
${ }^{5}$ The limits were calculated for the case of zero observed events in the GAPS and BESS experiments and in the TOF (lowenergy) region of the AMS-02 experiment. In the higher-energy $\mathrm{RICH}$ region of the AMS-02 experiment, one observed event was assumed.
} 


\section{AMS-02}

The antideuteron analysis of AMS-02 has been described in detail in Ref. [42]; however, since this was published, the decision was made to replace the superconducting magnet of the experiment with a permanent magnet. The AMS collaboration has yet to publish a re-analysis using the new setup, and thus the best estimate possible will be to broadly follow the existing analysis.

We calculate the expected number of observed antideuteron events at AMS-02 through

$$
N_{\bar{d}}=\int A_{\bar{d}} C_{\mathrm{Geo}} t \Phi_{\bar{d}} d T,
$$

where $t$ is the exposure time, $\Phi_{\bar{d}}$ is the antideuteron flux outside the Earth's magnetosphere, $A_{\bar{d}}$ is the antideuteron acceptance with selection cuts taken into account, and $C_{\mathrm{Geo}}$ is the geomagnetic transmission function (GTF), which accounts for the drop in low-energy charged particle fluxes due to the geomagnetic field. The integral is performed over the sensitivity range(s) of the experiment. AMS-02 has two relevant sensitivity ranges, $0.2-0.8 \mathrm{GeV} / \mathrm{n}$ and 2.2-4.2 GeV/n, corresponding to the Time-of-Flight (TOF) detector and the Ring Imaging Cherenkov Counter (RICH), respectively. We calculate the expected number of events separately for the two energy ranges.

As in the original analysis, we assume an exposure time of 3 years. The acceptance, $A_{\bar{d}}$, after selection cuts, as a function of the kinetic energy, is taken from Fig. 2 in Ref. [16]. For the geomagnetic transmission function, $C_{\mathrm{Geo}}$, we use the CREME96 [43] precalculated GTF for the International Space Station orbit in quiet geomagnetic weather conditions, as shown in Fig. 6, as a function of particle rigidity.

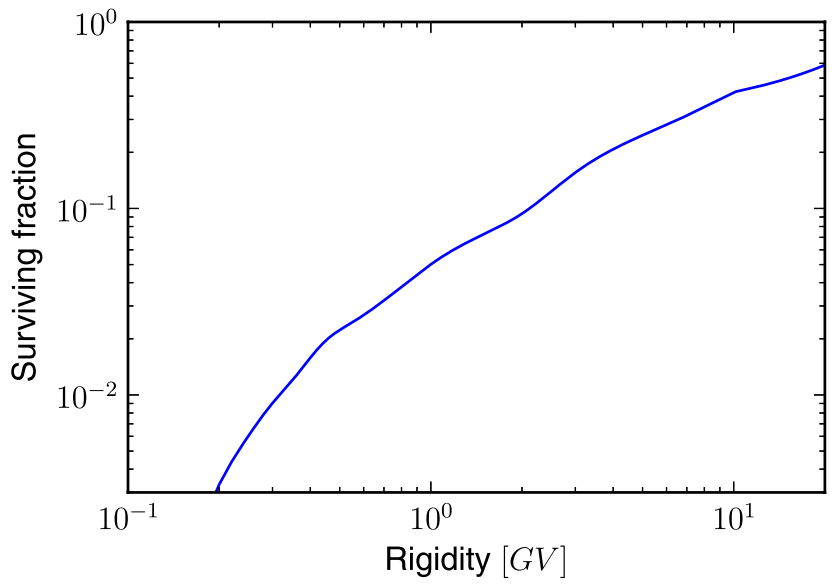

FIG. 6 (color online). Geomagnetic transmission function for the International Space Station orbit in quiet geomagnetic weather conditions. The GTF gives the surviving fraction of incident charged particles as a function of their rigidity.
We note that this GTF yields a more conservative result than the GTF used in the original analysis. For comparison, the antideuteron flux from DM model b) in Ref. [42] was reported to yield two expected events in the TOF region with 3 years of exposure, while in our calculation this model only yields 1.2 events.

In the BESS experiments, no events were observed, and the expected number of background events is not needed for setting exclusion limits with the $C L_{s}$ method. If an event is observed, however, the expected number of background events is needed. The number of astrophysical antideuteron background events for AMS-02 can be calculated directly from Eq. (20). However, the main source of background for AMS-02 is misreconstructed (anti)protons, deuterons and electrons. We estimate the number of such background events using

$$
N_{\mathrm{BG}}=\sum_{i} \int \frac{A_{i}}{R_{i}} C_{\mathrm{Geo}} t \Phi_{i} d T
$$

where $\Phi_{i}$ and $A_{i}$ are the flux and acceptance for particle species $i$, respectively, and $R_{i}$ is the corresponding rejection factor.

The rejection factor for a given particle species is defined as the ratio between the number of particles surviving the selection cuts and the number of particles (wrongfully) identified as antideuterons. The rejection factors are in principle energy dependent, but only averaged rejection factors are publicly available. The full list of rejection factors, taken from Ref. [42], is found in Table VI.

The acceptances for the background species after antideuteron selection cuts, $A_{i}$, have not been released (except for deuterons), and in our analysis we therefore use the signal (antideuteron) acceptance $A_{\bar{d}}$ as a conservative estimate. For the fluxes of the background species, we use the fits to experimental data compiled in Fig. 2.4 of Ref. [44].

Our calculation yields an expected background of 0.05 events in the TOF sensitivity range and 0.63 events in the RICH sensitivity range in the 3-year exposure. When we later calculate prospective limits from AMS-02, we assume zero observed events in the TOF range and one observed event in the RICH range; this is in order to represent the most likely background-only observation.

TABLE VI. AMS-02 rejection factors for antiprotons, protons, deuterons and electrons in the TOF and RICH energy ranges.

\begin{tabular}{lccc}
\hline \hline Rejection Factor & TOF Range & \multicolumn{2}{c}{ RICH Range } \\
\hline$R_{\bar{p}}$ & $6 \times 10^{6}$ & $2 \times 10^{5}$ & $T<7.2 \mathrm{GeV}$ \\
& $2 \times 10^{4}$ & $7.2<T<8.9 \mathrm{GeV}$ \\
$R_{p}$ & $8 \times 10^{11}$ & $8 \times 10^{11}$ & \\
$R_{d}$ & $1.5 \times 10^{9}$ & $1.5 \times 10^{9}$ & \\
$R_{e}$ & $2 \times 10^{9}$ & $5 \times 10^{7}$ & \\
\hline \hline
\end{tabular}




\section{GAPS}

There is currently not enough information available in order to make an analysis for GAPS that is as thorough as the AMS-02 analysis. In order to estimate the number of expected events at GAPS, we will instead follow the approach of Ref. [45], where an effective exposure $\langle\mathcal{E}\rangle$ is estimated from

$$
D=\int \Phi(E) \mathcal{E}(E) d E \simeq \Phi_{\Delta E} \times\langle\mathcal{E}\rangle \times \Delta E
$$

where $D$ is the expected number of signal events, $\mathcal{E}(E)$ is the true, energy-dependent detector exposure, and $\Phi_{\Delta E}$ is the expected flux in the energy interval $\Delta E$. For the GAPS Long Duration Balloon flight $(\mathrm{LDB}+)$, $D=1$ expected events correspond to a flux of $\Phi_{\Delta E}=2.8 \times 10^{-7}\left(\mathrm{~m}^{2} \mathrm{~s} \mathrm{sr} \mathrm{GeV} / \mathrm{n}\right)^{-1}$ in the energy interval $0.1-0.25 \mathrm{GeV} / \mathrm{n}$ [45], thus giving an effective exposure of $\langle\mathcal{E}\rangle=2.36 \times 10^{7} \mathrm{~m}^{2} \mathrm{~s} \mathrm{sr}$.

Using this estimated effective exposure, we can use Eq. (22) to find the expected number of signal events for any other antideuteron flux. Unfortunately, we do not have an estimate for the number of background events, and we can therefore only set limits for the case of zero observed events.

\section{LIMITS ON RPV COUPLINGS}

With the expected signal and background fluxes in hand we can now proceed to setting limits on the RPV couplings and gravitino masses. We use the $C L_{s}$ method [46,47] because of its robustness in the face of low backgrounds with possible downward fluctuations.

In this calculation, the NFW DM halo profile and "med" propagation parameters are assumed to be correct, and we have focused on the uncertainties in antideuteron production. Ideally, the uncertainties related to the propagation and the DM distribution should also be taken into account in the $C L_{s}$ calculation. These uncertainties are, however, difficult to estimate with our current knowledge, and in the literature they are typically handled by giving upper and lower limits corresponding to the "max" and "min" propagation models and extremal halo profile cases. The antideuteron fluxes from the "min" and "max" models typically differ by $\sim 2$ orders of magnitude, with the "med" model lying somewhere in between-more or less in the middle for kinetic energies $T \sim 10^{-1} \mathrm{GeV} / \mathrm{n}$, and increasingly closer to the "max" model for increasing energies; see e.g. Ref. [20].

\section{A. The $C L_{s}$ method}

Given a test statistic $X$ that is constructed to increase monotonically for increasingly signal-like events, the $C L_{s}$ confidence is given by

$$
C L_{s}=\frac{P_{s+b}\left(X \leq X_{\mathrm{obs}}\right)}{P_{b}\left(X \leq X_{\mathrm{obs}}\right)},
$$

where $X_{\mathrm{obs}}$ is the value of the test statistic measured by an experiment, and $P_{i}\left(X \leq X_{\mathrm{obs}}\right)$ is the probability of having $X \leq X_{\text {obs }}$ given that hypothesis $i$ is true. The subscripts $b$ and $s+b$ correspond to the background and signal+ background hypotheses, respectively. A signal can be considered excluded at a confidence level $C L$ when

$$
1-C L_{s} \geq C L \text {. }
$$

We calculate the $C L_{s}$ limits using the best-fit point in $p_{0}$ and the hadronization parameters, given in Sec. II B 4, henceforth referred to as the nuisance parameters. For an assumed value of the RPV coupling $\lambda$ and gravitino mass $m_{\tilde{G}}$, we use the test statistic

$$
Q\left(\lambda, m_{\tilde{G}}\right)=e^{-s_{\text {tot }}} \prod_{i=1}^{N_{\text {chan }}}\left(1+\frac{s_{i}}{b_{i}}\right)^{n_{i}}
$$

where the product is taken over all bins and channels of an experiment, $s_{i}=s_{i}\left(\lambda, m_{\tilde{G}}\right)$ and $b_{i}$ are the expected number of signal and background events in bin $i, s_{\text {tot }}=\sum_{i} s_{i}$ is the expected total number of signal events, and $n_{i}$ is the observed number of events in bin $i$.

Ideally, one should also include the nuisance parameter uncertainties in the calculation, as these tend to weaken the limits somewhat. Instead of using $Q\left(\lambda, m_{\tilde{G}}\right)$ one would then use a test statistic such as the profile likelihood ratio,

$$
q\left(\lambda, m_{\tilde{G}}\right)=\frac{\mathcal{L}\left(\lambda, m_{\tilde{G}}, \hat{\hat{\theta}}_{i}\right)}{\mathcal{L}\left(\hat{\lambda}, \hat{m}_{\tilde{G}}, \hat{\theta}_{i}\right)},
$$

formed from the ratio of the likelihood for the choice of $\lambda$ and $m_{\tilde{G}}$ and the corresponding best-fit values of the nuisance parameters $\hat{\hat{\theta}}_{i}$, and the likelihood of the best-fit point for $\lambda, m_{\tilde{G}}$ and the nuisance parameters $\theta_{i}$. The relevant likelihood would then be

$$
\mathcal{L}\left(\lambda, m_{\tilde{G}}, \theta_{i}\right)=\mathcal{L}_{\text {cosmic }}\left(\lambda, m_{\tilde{G}}, \theta_{i}\right) \cdot \mathcal{L}_{\text {collider }}\left(\theta_{i}\right),
$$

where $\mathcal{L}_{\text {collider }}\left(\theta_{i}\right)$ is the likelihood function corresponding to the $\chi^{2}$ distribution found in our tuning of the nuisance parameters against collider experiments, while $\mathcal{L}_{\text {cosmic }}\left(\lambda, m_{\tilde{G}}, \theta_{i}\right)$ is the likelihood for a given observation at one of the cosmic-ray experiments for the particular values of $\lambda, m_{\tilde{G}}$, and $\theta_{i}$.

Unfortunately, the likelihood maximization is too computationally expensive to perform for every gravitino mass and coupling as it involves generating large samples of antideuterons for many different nuisance parameters, where each sample takes days on a single CPU due to the inefficiencies in the per-event coalescence model. From 
a test based on the $\bar{U}_{1} \bar{D}_{1} \bar{D}_{2}$ operator for a specific gravitino mass of $200 \mathrm{GeV}$, we estimate that including the nuisance parameter uncertainties can weaken the limits we set by approximately $20 \%$ in the experiments studied here.

\section{Limits with single-bin counting experiments}

For single-bin counting experiments such as BESS and GAPS, the signal exclusion confidence in the $C L_{s}$ scheme can be simplified to [47]

$$
C L=1-\frac{\sum_{n=0}^{n_{\mathrm{obs}}} \frac{e^{-(b+s)}(b+s)^{n}}{n !}}{\sum_{n=0}^{n_{\text {obs }} \frac{e^{-b} b^{n}}{n !}},}
$$

where $s$ and $b$ are the expected number of signal and background events, respectively, and $n_{\mathrm{obs}}$ is the number of observed events. For the case of zero observed events, which we assume here, this expression further simplifies to

$$
C L=1-e^{-s},
$$

which is independent of the background expectation.

\section{Setting $C L_{s}$ limits for $A M S-02$}

For AMS-02, we have two bins, corresponding to the TOF and RICH detector sensitivity regions. The background expectations $b_{1}$ and $b_{2}$ for these detectors are known, and given a value for the RPV coupling in question, we can find the corresponding signal expectations, $s_{1}$ and $s_{2}$. We assume $n_{1}=0$ observed events in the TOF detector and $n_{2}=1$ observed event in the RICH detector, and calculate $Q_{\mathrm{obs}}$ according to Eq. (25).

We then proceed by calculating $P_{s+b}\left(Q \leq Q_{\text {obs }}\right)$ and $P_{b}\left(Q \leq Q_{\text {obs }}\right)$. This is done by finding all pairs of $\left(n_{1}, n_{2}\right)$ such that the corresponding value of $Q$ calculated from Eq. (25) fulfills $Q \leq Q_{\text {obs }}$. We denote this set of pairs $\mathcal{C}$. Assuming first that the signal+background hypothesis is true, and that the number of events in bin $i$ follows a Poisson distribution with expectation value $s_{i}+b_{i}$, the probability of observing exactly $n_{1}$ and $n_{2}$ events at TOF and RICH, respectively, is given by a product of two Poisson distributions,

$$
P_{s+b}\left(n_{1}, n_{2}\right)=\frac{\left(s_{1}+b_{1}\right)^{n_{1}}}{n_{1} !} e^{-\left(s_{1}+b_{1}\right)} \frac{\left(s_{2}+b_{2}\right)^{n_{2}}}{n_{2} !} e^{-\left(s_{2}+b_{2}\right)} .
$$

The probability $P_{s+b}\left(Q \leq Q_{\text {obs }}\right)$ is then given by the sum of all probabilities $P_{s+b}\left(n_{1}, n_{2}\right)$ where $\left(n_{1}, n_{2}\right)$ satisfies $Q \leq Q_{\text {obs }}$, i.e.

$$
P_{s+b}\left(Q \leq Q_{\mathrm{obs}}\right)=\sum_{\left(n_{1}, n_{2}\right) \in \mathcal{C}} P_{s+b}\left(n_{1}, n_{2}\right) .
$$

The procedure for calculating $P_{b}\left(Q \leq Q_{\mathrm{obs}}\right)$ is identical, but it uses $s_{1}=s_{2}=0$ in Eq. (30). Inserting these results into Eq. (23), we then find the $C L_{s}$ confidence value for the given value of the coupling.

The 95\% $C L_{s}$ confidence limit on the flux or coupling is finally found by varying the flux or coupling until the value for which $C L_{s}=1-0.95$ is found.

\section{B. Current BESS limits}

Figure 7 shows the limits we can currently set on various RPV couplings from the nonobservation of antideuterons by BESS. We see that, as expected, overall limits on the $\bar{U} \bar{D} \bar{D}$ couplings are stronger than for $L Q \bar{D}$, although some of this effect is, as pointed out above, from the shorter lifetime of the gravitino, and not directly connected to the (anti)quark content of the operator.

The limits on the $L_{1} Q_{1} \bar{D}_{2}$ and $\bar{U}_{1} \bar{D}_{1} \bar{D}_{2}$ operators from antideuterons at BESS are somewhat weaker than the existing limits found using antiproton data from PAMELA in Ref. [13]. For the $L_{1} Q_{3} \bar{D}_{3}$ and $\bar{U}_{3} \bar{D}_{2} \bar{D}_{3}$ operators, the limits disappear when reaching the kinematical thresholds of the corresponding gravitino three-body decays.

The reader is warned to keep in mind that the antiproton limits in Ref. [13] were calculated using PYTHIA 6.4 for event generation and GALPROP for cosmic-ray propagation, with somewhat different propagation model parameters than used in the present study. Differences in the hadronization and propagation model can lead to differences in limits, and this should be kept in mind when comparing the antiproton and antideuteron limits.

\section{Expected AMS-02 limit}

In Fig. 8 we show the expected achievable limit on the same RPV couplings from the AMS-02 experiment under the assumption of one observed event, consistent with the expected background. We see an overall significant

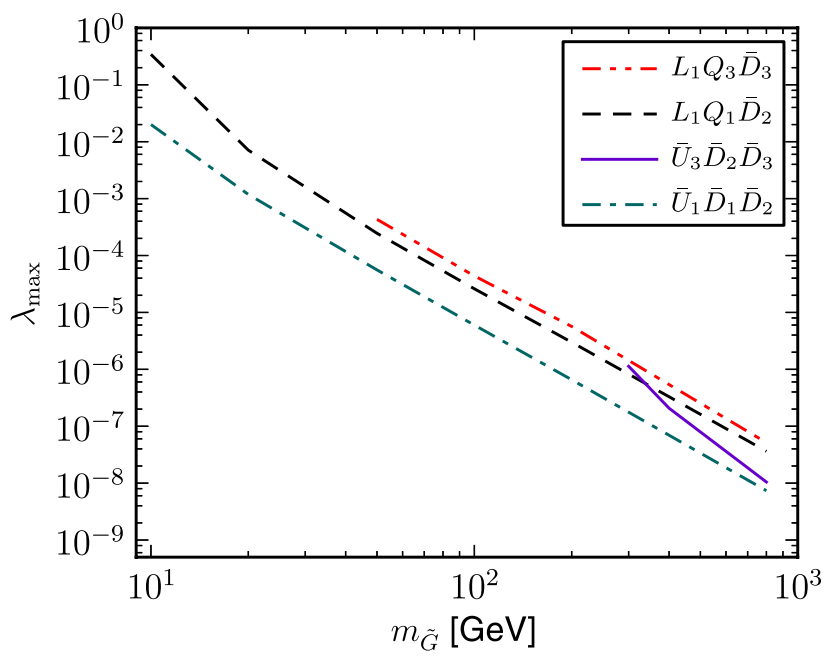

FIG. 7 (color online). Upper limits on various RPV couplings $\lambda$ as a function of gravitino mass from BESS antideuteron searches. The colors show different RPV couplings. All limits are for the "med" propagation model and NFW DM halo profile. 


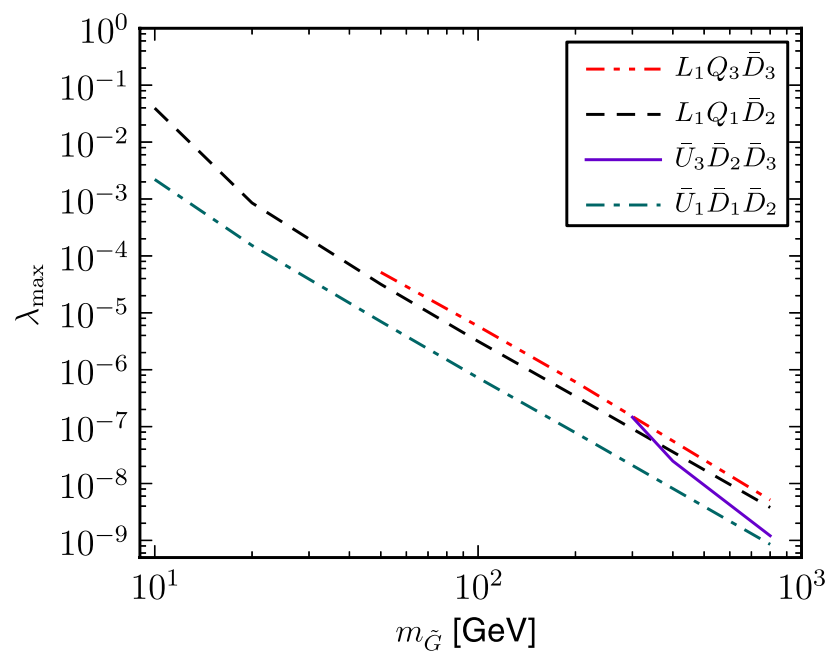

FIG. 8 (color online). Expected achievable upper limits on various RPV couplings $\lambda$ as a function of gravitino mass from the AMS-02 experiment. The limit assumes zero and one event observed in the TOF and RICH energy ranges, respectively. The colors show different RPV couplings. All limits are for the "med" propagation model and NFW DM halo profile.

strengthening of the bounds compared to the bounds from BESS. The expected limit on the $L_{1} Q_{2} \bar{D}_{2}$ operator is somewhat weaker than the current limit from antiprotons in Ref. [13] for low gravitino masses, but roughly equal for gravitino masses above a few hundred GeV. For the $\bar{U}_{1} \bar{D}_{1} \bar{D}_{2}$ operator, the expected limit from AMS-02 is a factor $\sim 1.5$ stronger than the current limit for $m_{\tilde{G}}=10 \mathrm{GeV}$, increasing to a factor $\sim 4$ for gravitino masses in the $100 \mathrm{GeV}$ range.

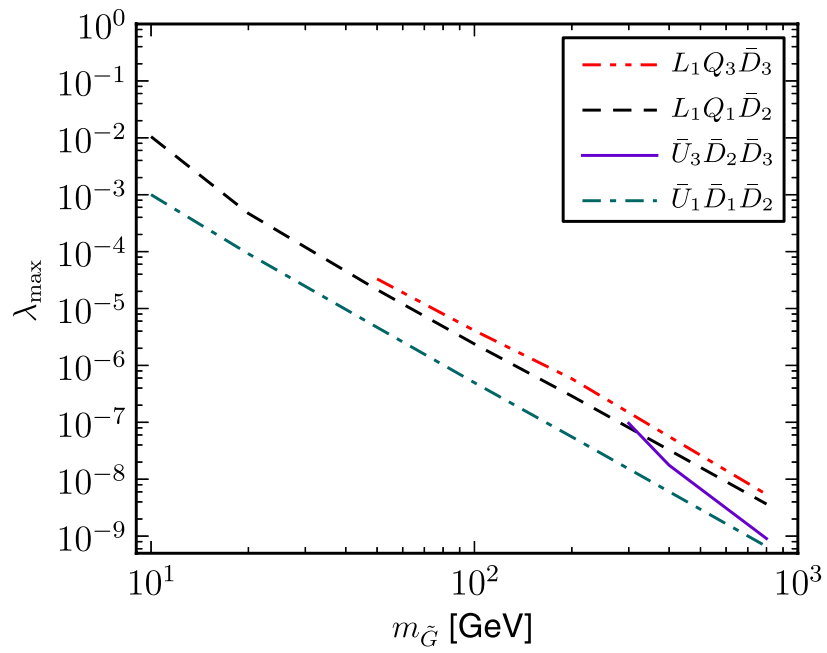

FIG. 9 (color online). Expected achievable upper limits on various RPV couplings $\lambda$ as a function of gravitino mass from the LDB + flight of the GAPS experiment. The limit assumes zero events observed. The colors show different RPV couplings. All limits are for the "med" propagation model and NFW DM halo profile.

\section{Expected GAPS limit}

The prospective limits on RPV couplings from the GAPS experiment are shown in Fig. 9. We see that the expected limits on the RPV couplings from GAPS are a factor 2-4 stronger than the expected limits from AMS-02 for the lowest gravitino masses, but are roughly equal for gravitino masses approaching $800 \mathrm{GeV}$. This is not unexpected, as the GAPS sensitivity range lies at lower energies than the AMS-02 ranges, and the peak in the antideuteron spectrum moves towards higher energies with increasing gravitino masses.

\section{CONCLUSIONS}

In this paper we have studied the influence of coalescence and hadronization parameters on the yield of antideuterons in dark matter decay/annihilation. By fitting to relevant collider data we provide a recommended set of parameter values for the HERWIG++ event generator in Table IV, with corresponding uncertainties that can be used for error propagation.

With the tuned formation model we have set bounds on trilinear RPV couplings in scenarios with gravitino dark matter using the results of antideuteron searches with the BESS experiment. These are of the same order of magnitude as limits from antiproton searches with PAMELA. We find large differences between different types of operators: the antideuteron searches are far more constraining - an order of magnitude-for the baryon-number-violating operators, and are more constraining for operators with light quarks. We have further investigated the potential of AMS-02 and GAPS to improve on these limits, and found that future results can strengthen current limits by a factor of $\sim 4$ for the baryon-number-violating operators in the whole range of gravitino masses considered (10-800 GeV). This will significantly improve on the current best limits for trilinear RPV couplings that come from antiproton searches.

\section{ACKNOWLEDGMENTS}

L. A. D. and A.R.R. would like to thank Andreas Papaefstathiou for valuable assistance with the internal workings of HERWIG++ and moral support, and Philip von Doetinchem for clarifying details in the calculation of expected events in the experiments. We would also like to express our gratitude to Alex Read for his patience in explaining statistics to theorists. The CPU-intensive parts of this work were performed on the Abel Cluster, owned by the University of Oslo and the Norwegian Metacenter for High Performance Computing (NOTUR), and operated by the Research Computing Services group at USIT, the University of Oslo IT Department. The computing time was given by NOTUR allocation NN9284K financed through the Research Council of Norway. 
[1] F. Takayama and M. Yamaguchi, Phys. Lett. B 485, 388 (2000).

[2] W. Buchmuller, L. Covi, K. Hamaguchi, A. Ibarra, and T. Yanagida, J. High Energy Phys. 03 (2007) 037.

[3] S. Lola, P. Osland, and A. R. Raklev, Phys. Lett. B 656, 83 (2007).

[4] F. Donato, N. Fornengo, and P. Salati, Phys. Rev. D 62, 043003 (2000).

[5] A. Ibarra and D. Tran, J. Cosmol. Astropart. Phys. 06 (2009) 004.

[6] M. Grefe, arXiv:1111.6779.

[7] M. Bahr et al., Eur. Phys. J. C 58, 639 (2008).

[8] K. Arnold et al., arXiv:1205.4902.

[9] P. Fayet, Phys. Lett. 69B, 489 (1977).

[10] L. E. Ibanez and G. G. Ross, Phys. Lett. B 260, 291 (1991).

[11] L. E. Ibanez and G. G. Ross, Nucl. Phys. B368, 3 (1992).

[12] S. Lola and G. G. Ross, Phys. Lett. B 314, 336 (1993).

[13] N.-E. Bomark, S. Lola, P. Osland, and A. R. Raklev, Phys. Lett. B 686, 152 (2010).

[14] A. Ibarra and S. Wild, J. Cosmol. Astropart. Phys. 02 (2013) 021.

[15] H. Fuke et al., Phys. Rev. Lett. 95, 081101 (2005).

[16] V. Choutko and F. Giovacchini on behalf of the AMS Collaboration, Proceedings of the 30th International Cosmic Ray Conference, Merida, Yucatan, Mexico, 2007, edited by R. Caballero, J. C. DOlivo, G. Medina-Tanco, L. Nellen, F. A. Sánchez, and J. F. Valdés-Galicia (Universidad Nacional Autónoma de México, Mexico City, Mexico, 2008), p. 765.

[17] J. E. Koglin et al., J. Phys. Conf. Ser. 120, 042011 (2008).

[18] G. Moreau and M. Chemtob, Phys. Rev. D 65, 024033 (2001).

[19] M. Kadastik, M. Raidal, and A. Strumia, Phys. Lett. B 683, 248 (2010).

[20] L. A. Dal and M. Kachelriess, Phys. Rev. D 86, 103536 (2012).

[21] L. A. Dal, Master's thesis, Norwegian University of Science and Technology, 2011, http://www.urn.kb.se/resolve? urn=urn:nbn:no:ntnu:diva-12634.

[22] S. Schael et al. (ALEPH Collaboration), Phys. Lett. B 639, 192 (2006).

[23] T. Sjostrand, S. Mrenna, and P. Z. Skands, J. High Energy Phys. 05 (2006) 026.

[24] T. Sjostrand, S. Mrenna, and P. Z. Skands, Comput. Phys. Commun. 178, 852 (2008).

[25] D. M. Asner et al. (CLEO Collaboration), Phys. Rev. D 75, 012009 (2007).
[26] S. Chekanov et al. (ZEUS Collaboration), Nucl. Phys. B786, 181 (2007).

[27] B. Alper et al., Phys. Lett. 46B, 265 (1973).

[28] S. Henning et al. (British-Scandinavian-MIT Collaboration), Lett. Nuovo Cimento 21, 189 (1978).

[29] N. Sharma et al. (ALICE Collaboration), Acta Phys. Pol. B Proc. Suppl. 5, 605 (2012).

[30] P. Richardson and D. Winn, Eur. Phys. J. C 72, 2178 (2012).

[31] R. Akers et al. (OPAL Collaboration), Z. Phys. C 63, 181 (1994).

[32] D. Buskulic et al. (ALEPH Collaboration), Z. Phys. C 66, 355 (1995).

[33] F. James and M. Roos, Comput. Phys. Commun. 10, 343 (1975).

[34] M. D. Buhmann, Radial Basis Functions: Theory and Implementations (Cambridge University Press, Cambridge, England, 2003).

[35] A. Ibarra and S. Wild, Phys. Rev. D 88, 023014 (2013).

[36] A. Baldini, V. Flaminio, W. G. Moorhead, and D. R. O. Morrison, in Landolt-Börnstein-Group I Elementary Particles, Nuclei and Atoms Vol. 12b, edited by H. Schopper (Springer, Berlin, 1988), http://www .springermaterials.com/docs/info/10367917_24.html.

[37] C. Amsler et al. (Particle Data Group Collaboration), Phys. Lett. B 667, 1 (2008).

[38] J. F. Navarro, C. S. Frenk, and S. D. M. White, Astrophys. J. 462, 563 (1996).

[39] F. Donato, N. Fornengo, D. Maurin, and P. Salati, Phys. Rev. D 69, 063501 (2004).

[40] F. Donato, D. Maurin, P. Salati, A. Barrau, G. Boudoul, and R. Taillet, Astrophys. J. 563, 172 (2001).

[41] L. J. Gleeson and W. I. Axford, Astrophys. J. 154, 1011 (1968).

[42] F. Giovacchini, Ph.D. thesis, Universitá di Bologna, Italy, 2007.

[43] A. J. Tylka, J. H. Adams, P. R. Boberg, B. Brownstein, W. F. Dietrich, E. O. Flueckiger, E. L. Petersen, M. A. Shea, D. F. Smart, and E. C. Smith, IEEE Trans. Nucl. Sci. 44, 2150 (1997).

[44] P. von Doetinchem, H. Gast, and S. Schael, arXiv: 0906.1070.

[45] N. Fornengo, L. Maccione, and A. Vittino, J. Cosmol. Astropart. Phys. 09 (2013) 031.

[46] A. L. Read, J. Phys. G 28, 2693 (2002).

[47] A. L. Read, in 1st Workshop on Confidence Limits, CERN, Geneva, Switzerland, 2000, edited by L. Lyons, Y. Perrin, and F. E. James (CERN, Geneva, 2000), p. 81. 